\title{
Thermal noise limit for ultra-high vacuum noncontact atomic force microscopy
}

\author{
Jannis Lübbe ${ }^{1}$, Matthias Temmen ${ }^{1}$, Sebastian Rode ${ }^{2,3}$, Philipp Rahe ${ }^{2,4}$, \\ Angelika Kühnle ${ }^{2}$ and Michael Reichling ${ }^{* 1}$
}

\section{Full Research Paper}

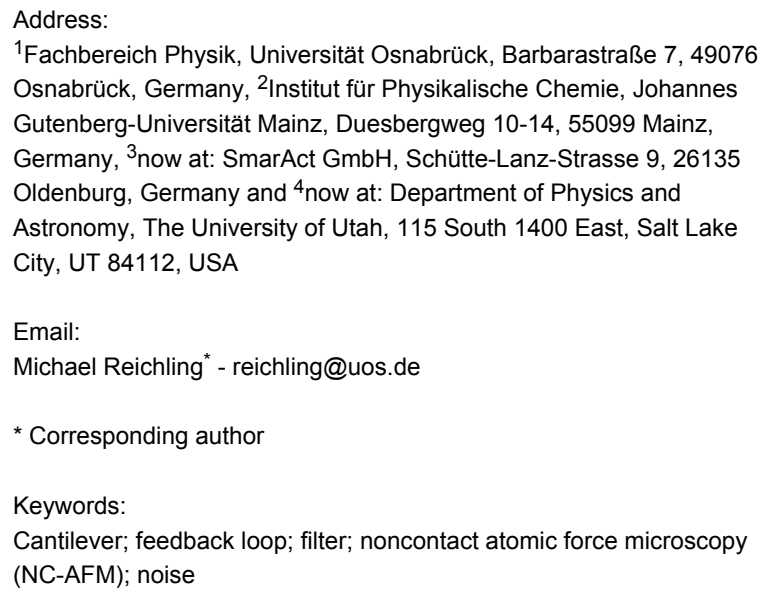

${ }^{1}$ Fachbereich Physik, Universität Osnabrück, Barbarastraße 7, 49076 Osnabrück, Germany, ${ }^{2}$ Institut für Physikalische Chemie, Johannes Gutenberg-Universität Mainz, Duesbergweg 10-14, 55099 Mainz, Germany, ${ }^{3}$ now at: SmarAct GmbH, Schütte-Lanz-Strasse 9, 26135 Oldenburg, Germany and ${ }^{4}$ now at: Department of Physics and Astronomy, The University of Utah, 115 South 1400 East, Salt Lake City, UT 84112, USA

Email:

Michael Reichling* - reichling@uos.de

* Corresponding author

Keywords:

Cantilever; feedback loop; filter; noncontact atomic force microscopy (NC-AFM); noise

Beilstein J. Nanotechnol. 2013, 4, 32-44.

doi:10.3762/bjnano.4.4

Received: 04 October 2012

Accepted: 14 December 2012

Published: 17 January 2013

This article is part of the Thematic Series "Advanced atomic force microscopy techniques".

Guest Editors: T. Glatzel and U. D. Schwarz

(C) 2013 Lübbe et al; licensee Beilstein-Institut. License and terms: see end of document.

\begin{abstract}
The noise of the frequency-shift signal $\Delta f$ in noncontact atomic force microscopy (NC-AFM) consists of cantilever thermal noise, tip-surface-interaction noise and instrumental noise from the detection and signal processing systems. We investigate how the displacement-noise spectral density $d^{z}$ at the input of the frequency demodulator propagates to the frequency-shift-noise spectral density $d^{\Delta f}$ at the demodulator output in dependence of cantilever properties and settings of the signal processing electronics in the limit of a negligible tip-surface interaction and a measurement under ultrahigh-vacuum conditions. For a quantification of the noise figures, we calibrate the cantilever displacement signal and determine the transfer function of the signal-processing electronics. From the transfer function and the measured $d^{z}$, we predict $d^{\Delta f}$ for specific filter settings, a given level of detection-system noise spectral density $d_{\mathrm{ds}}^{z}$ and the cantilever-thermal-noise spectral density $d^{z}$ th. We find an excellent agreement between the calculated and measured values for $d^{\Delta f}$. Furthermore, we demonstrate that thermal noise in $d^{\Delta f}$, defining the ultimate limit in NC-AFM signal detection, can be kept low by a proper choice of the cantilever whereby its $Q$-factor should be given most attention. A system with a low-noise signal detection and a suitable cantilever, operated with appropriate filter and feedback-loop settings allows room temperature NC-AFM measurements at a low thermal-noise limit with a significant bandwidth.
\end{abstract}

\section{Introduction}

In this contribution, we discuss noise in frequency-modulation noncontact atomic force microscopy (NC-AFM) using cantilevers as force sensors and optical beam deflection (OBD) for signal detection. Figure 1 shows a schematic diagram of an NC-AFM setup based on OBD to illustrate the signal path and the quantities describing noise. Measured quantities discussed 


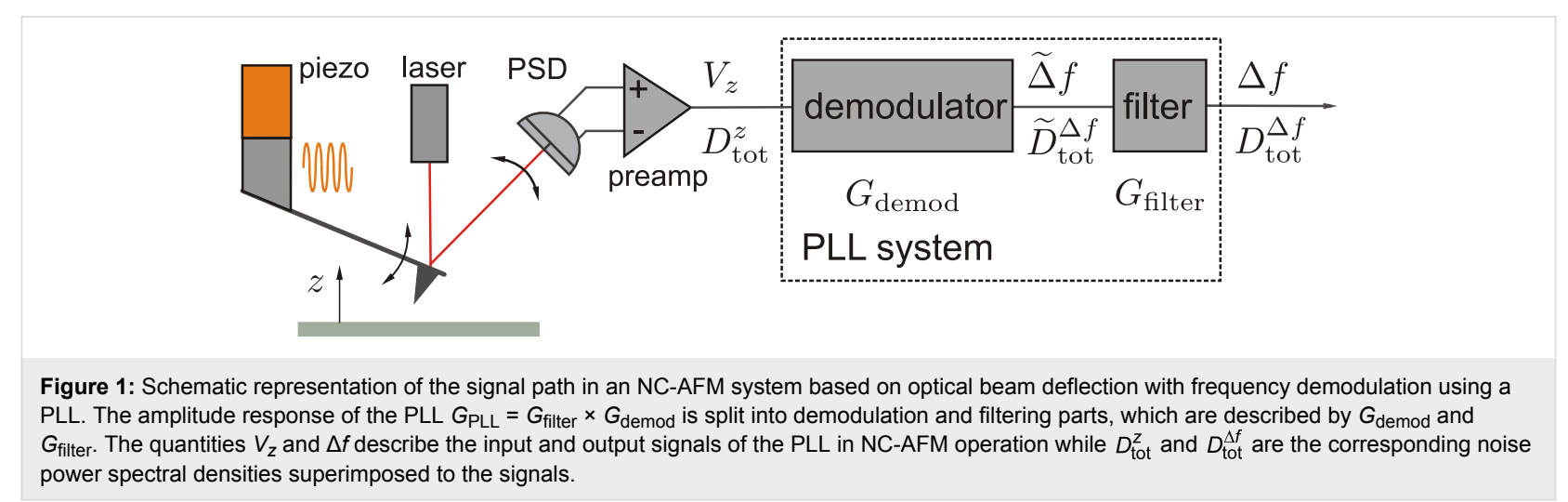

here are often electrical signals that are equivalent to quantities describing the mechanical oscillation of the cantilever. The calibration procedure described in Section 1 of Supporting Information File 1 establishes a relation between the representation in mechanical and electrical units. During NC-AFM operation, the cantilever with eigenfrequency $f_{0}$ is excited to oscillation at the resonance frequency $f_{r}$, which differs from its eigenfrequency by the frequency shift $\Delta f=f_{r}-f_{0}$ when there is a tip-surface interaction. The mechanical oscillation, i.e., a periodic displacement $z(t)$ of the cantilever with amplitude $A$, is converted into the oscillation signal $V_{z}(t)$ by the position-sensitive detector (PSD) connected to the preamplifier. The amplitude $A$ of this signal is determined and stabilised to a preset value by the amplitude feedback loop. Signal processing in NC-AFM involves the demodulation of the periodic cantilever-displacement signal $V_{z}(t)$ as well as filtering in the frequency domain to yield the frequency shift $\Delta f(t)$ carrying the information on the tip-surface interaction [1]. Demodulation is commonly performed by a phase-locked loop (PLL) circuit [2]. As schematically depicted in Figure 1, the amplitude response of the PLL unit can formally be decomposed into the amplitude response $G_{\text {demod }}$ of the demodulator and the amplitude response $G_{\text {filter }}$ of an in-loop or output filter. The characteristics of $G_{\text {filter }}$ can be set by the user according to the needs of the experiment.

Noise in NC-AFM consists mainly of three contributions: noise arising from the thermal excitation of a cantilever or another force sensor, noise caused by the detection system and signal processing electronics $[3,4]$, and instabilities arising from the interaction of the force microscopy tip with the surface as well as arising from the feedback loops stabilising the cantilever oscillation amplitude and the tip-surface distance [5]. Here, we investigate noise for the case of negligible tip-surface interaction and discuss the cantilever-displacement thermal-noise spectral density $d_{\mathrm{th}}^{z}(f)$ as well as the displacement-equivalent noise spectral density $d_{d s}^{z}(f)$ introduced by the detection system. This is carried out here in search of the ultimate limits of detection defined by thermal noise, while a systematic study of the tip-sample interaction noise that is present in any NC-AFM imaging or spectroscopy experiment will be the subject of forthcoming work. Here, we entirely focus the discussion on cantilever-based NC-AFM; however, the concepts, theoretical framework, and experimental strategies for the noise analysis can easily be transferred to systems based on other force sensors and detection schemes.

Under ultrahigh-vacuum (UHV) conditions, the thermal noise of the cantilever is usually small compared to the noise of the detection system due to the high $Q$-factor of the cantilever in vacuum [6]. The instrumental noise sources in an optical beam deflection (OBD) setup were recently discussed in detail [3] and it was found that the major noise sources are shot noise arising from the photodetector as well as Johnson noise originating from the resistors in the preamplifier. Further noise is generated in the laser diode that is mainly quantum noise for small output power and mode-hopping noise for large output power [3]. Back reflections of the laser beam into the laser optical resonator may increase mode hopping. The laser spot on the photodiode may further be disturbed by optical interference, creating time-varying speckle patterns due to temperature fluctuations and mechanical instability. It has been shown, however, that by operating the laser diode with radio-frequency modulation, the contribution of the light source to the total noise can be reduced to a negligible minimum [3].

The issue of noise is intimately related to the requirements of the NC-AFM system to process signals varying in time. The detection bandwidth $B$ needed to retrieve the full information present in the $\Delta f(t)$ signal at the output of the PLL system depends on the spectral components produced during a scanning or spectroscopy experiment. Practically, the maximum usable bandwidth $B_{\max }$ is defined by the total displacementnoise spectral density $d_{\text {tot }}^{z}=\sqrt{\left(d_{\mathrm{th}}^{z}\right)^{2}+\left(d_{\mathrm{ds}}^{z}\right)^{2}}$ as schematically illustrated in Figure 2. In this figure, we show the displacement spectral density $d^{z}(f)$ present at the input of the frequency demodulator with contributions of the measurement signal and 


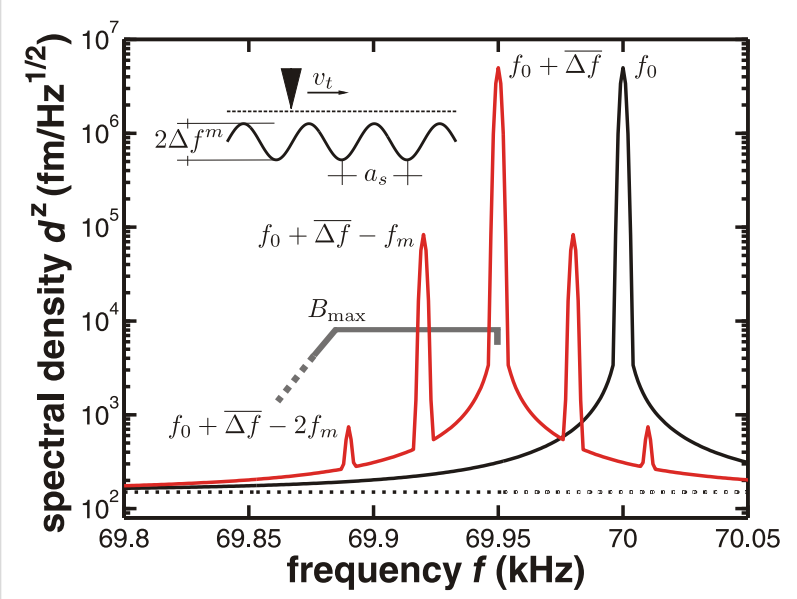

Figure 2: Illustrative representation for the spectral density of the displacement of a cantilever excited to oscillation with $10 \mathrm{~nm}$ amplitude at its eigenfrequency $f_{0}=70 \mathrm{kHz}$ without tip-sample interaction (black curve) and with tip-sample interaction resulting in a frequency modulation (red curve). Data is drawn for a modulation frequency of $f_{m}=30 \mathrm{~Hz}$, a modulation amplitude of $\Delta f^{m}=1 \mathrm{~Hz}$ and a mean frequency shift of $\overline{\Delta f}=-50 \mathrm{~Hz}$. A typical detection-system noise floor of $d_{\mathrm{ds}}^{z}=150 \mathrm{fm} / \sqrt{\mathrm{Hz}}$ (dotted line) as well as thermal noise based on the cantilever properties $\left(k=2.5 \mathrm{~N} / \mathrm{m}, Q_{0}=100000\right)$ are added to the signal. The inset schematically illustrates how scanning the tip over the sample having a spatial periodicity $a_{s}$ with a scan speed of $v_{t}$ yields a modulation at frequency $f_{m}=v_{t} / a_{s}$. The surface corrugation $\Delta z$ yields a modulation amplitude $\Delta f^{m}$ where the modulation index is $\Delta f^{m} / f_{m}=1 / 30$ for this example.

noise (see Figure 1) as a function of the frequency $f$. This quantity is the root of the one-sided power spectral density $D^{z}(f)$, which is derived from the displacement signal $V_{z}(t)$ via a Fourier transform as

$$
D^{z}(f)=\left(d^{z}(f)\right)^{2}=2\left|S \mathcal{F}\left\{V_{z}(t)\right\}(f)\right|^{2}
$$

where $S$ is the calibration factor converting voltage into displacement as defined in Section 1 of Supporting Information File 1 and $\mathcal{F}$ the Fourier transform of the displacement signal $V_{z}$ with:

$$
\mathcal{F}\left\{V_{z}(t)\right\}(f)=\int_{-\infty}^{\infty} V_{z}(t) \exp (-2 \pi i f t) d t
$$

For the case of absent tip-surface interaction, $d^{z}$ is a sharp peak centred at the cantilever eigenfrequency $f_{0}\left(f_{0}=70 \mathrm{kHz}\right.$ in Figure 2) including noise contributions from $d_{\mathrm{th}}^{z}$ and $d_{\mathrm{ds}}^{z}$, which will be described in detail below. In the presence of a tip-surface interaction, the resonance peak is shifted by the amount $\overline{\Delta f}(\overline{\Delta f}=-50 \mathrm{~Hz}$ in Figure 2$)$ caused by the timeinvariant part of the interaction. Additionally, sidebands appear that represent spectral components in $V_{z}(t)$ created during scanning or spectroscopy. For simplicity, we assume here a scan- ning of the tip over the surface with a speed $v_{t}$ where a periodic corrugation (period $a_{s}$ ) of the surface $\Delta f^{m}$ creates a sinusoidal modulation at the frequency $f_{m}=v_{t} / a_{s}\left(f_{m}=30 \mathrm{~Hz}\right.$ in Figure 2), i.e., $\Delta f(t)=\overline{\Delta f}+\Delta f^{m} \sin \left(2 \pi f_{m}+\varphi\right)$. Effectively, this is a frequency modulation of $V_{z}(t)$ with a modulation index $\Delta f^{m} / f_{m}$ producing an infinite number of higher harmonics with rapidly decreasing power [4]. How many of these side peaks can be detected depends on the modulation index of the signal and the noise characteristics of the measurement system. For the hypothetical measurement illustrated in Figure 2, only two sideband peaks are well above the noise floor. Here, the suitable bandwidth $B_{\max }$ is defined by the frequency of the second sideband peak.

The frequency demodulator extracts the frequency shift $\Delta f(t)$ from the periodic displacement signal $V_{z}(t)$ and, for an arbitrary signal, projects the power in the sidebands of $D^{z}(f)$ into the frequency-shift power spectral density $D^{\Delta f}\left(f_{m}\right)$, which can be represented as:

$$
\begin{gathered}
D^{\Delta f}\left(f_{m}\right)=\left(d^{\Delta f}\left(f_{m}\right)\right)^{2}=2\left|\mathcal{F}\{\Delta f(t)\}\left(f_{m}\right)\right|^{2} \\
\mathcal{F}\{\Delta f(t)\}\left(f_{m}\right)=\int_{-\infty}^{\infty} \Delta f(t) \exp \left(-2 \pi i f_{m} t\right) d t .
\end{gathered}
$$

The frequency shift $\Delta f(t)$ varies on a time scale that in an imaging experiment is determined by the spatial periodicity of the scanned structure and the scanning speed, rather than by the period of the cantilever oscillation. Therefore, the spectrum of the frequency shift signal present at the output of the demodulator has significant power only in a limited spectral range of $f_{m}$. The detection bandwidth $B$ of the demodulator is, therefore, usually restricted to a value of the order of $100 \mathrm{~Hz}$ to $1 \mathrm{kHz}$. As the noise is transformed by the demodulator in a similar way, we define $d_{\text {tot }}^{\Delta f}\left(f_{m}\right)$ and $D_{\text {tot }}^{\Delta f}\left(f_{m}\right)$ as the frequency-shift-noise spectral density and the frequency-shift-noise power spectral density, respectively, and discuss separate noise contributions $d_{\mathrm{th}}^{\Delta f}$ and $d_{\mathrm{ds}}^{\Delta f}$ to the frequency-shift signal $\Delta f$, as the noise contributions of the thermal cantilever excitation and the detection system yield different spectral characteristics. The detection bandwidth $B$ and, consequently, the noise propagation characteristics depend on the PLL amplitude response $G_{\mathrm{PLL}}=G_{\text {filter }}$ $\times G_{\text {demod }}$, which can usually be influenced by the operator through the filter settings (see Figure 1).

To understand the influence of various experimental parameters and the settings of the PLL filter on $D_{\text {tot }}^{\Delta f}\left(f_{m}\right)$, which is the most relevant noise figure in the NC-AFM experiment, we derive noise models based on system parameters. Hypotheses and conclusions are tested against the reality of NC-AFM 
experiments, by comparing the noise figures and filter settings for three NC-AFM systems based on the OBD scheme and comparing experimental results to the predicted settings for noise-optimised operation. We find that by the correct choice of the cantilever, by using optimised detection electronics and by appropriate PLL filter settings, the frequency-shift signal $\Delta f$ can be detected at a low thermal-noise limit over a bandwidth $B$ that is more than $100 \mathrm{~Hz}$ for room temperature operation under UHV conditions. The dependence of the thermal limit and other noise figures on relevant experimental parameters is discussed in detail.

\section{Displacement noise}

Here, we discuss the displacement noise superimposed on the displacement signal $V_{z}(t)$ in the case of negligible tip-surface interaction. Usually, the signal $V_{z}(t)$ is a noisy sinusoidally oscillating voltage and the noise can be described in the frequency domain by the displacement-noise spectral density $d_{\text {tot }}^{z}(f)$. This is the square root of the displacement-noise power spectral density $D_{\text {tot }}^{z}(f)$, which is proportional to the unwanted energy per frequency interval stored in the oscillating system.

A cantilever that is not deliberately excited but in equilibrium with a thermal bath at temperature $T$ exhibits random fluctuations resulting in measurable noise in the cantilever displacement signal. This noise can be predicted by a model outlined in Section 2 of Supporting Information File 1. Furthermore, all electrical and optical components that are part of the detection system produce noise, superimposed on the displacement signal. Therefore, the power spectral density of the total displacement signal noise $D_{\text {tot }}^{z}$ can be described as

$$
D_{\mathrm{tot}}^{z}=D_{\mathrm{th}}^{z}+D_{\mathrm{ds}}^{z}
$$

where $D_{\mathrm{th}}^{z}$ and $D_{\mathrm{ds}}^{z}$ represent the thermal and the detectionsystem contributions. The quantity $D_{\text {th }}^{z}(f)$ as derived in Section 2 of Supporting Information File 1 can be represented as:

$$
D_{\mathrm{th}}^{z}=\frac{2 k_{\mathrm{B}} T /\left(\pi k_{0} f_{0} Q_{0}\right)}{\left(1-\left(f / f_{0}\right)^{2}\right)^{2}+\left(f /\left(f_{0} Q_{0}\right)\right)^{2}} .
$$

Here, $D_{\text {th }}^{z}$ is calculated only for the fundamental cantilever oscillation mode with eigenfrequency $f_{0}$, stiffness $k_{0}$ and $Q$-factor $Q_{0}$ as the contribution of higher harmonics to the total noise power spectral density is small; the fundamental mode contains $97 \%$ or more of the total power extracted by the cantilever from the thermal bath. For the investigation of noise at higher harmonics, $f_{0}, k_{0}$ and $Q_{0}$ would have to be replaced by the respective modal values $f_{n}, k_{n}$ and $Q_{n}$ (see Section 2 of Supporting Information File 1). The noise spectral density $d_{\text {tot }}^{z}(f)$ as defined in Equation 1 is displayed in Figure 3a for typical experimental parameters exhibiting a sharp peak at $f_{0}=$ $70 \mathrm{kHz}$ (note the logarithmic scale of the ordinate) sticking far out of the white-noise floor $d_{\mathrm{ds}}^{z}(f)$ when using low-noise detection electronics. The sharp peak in $d_{\mathrm{th}}^{z}(f)$ results from the cantilever resonance. Especially high $Q$-factor cantilevers strongly amplify the white spectral power of thermal excitation only in a narrow range of frequencies around $f_{0}$ according to Equation 2. The detection-system noise represented by $d_{\mathrm{ds}}^{z}(f)$ is governed by the quality of the optical and electronic compo-
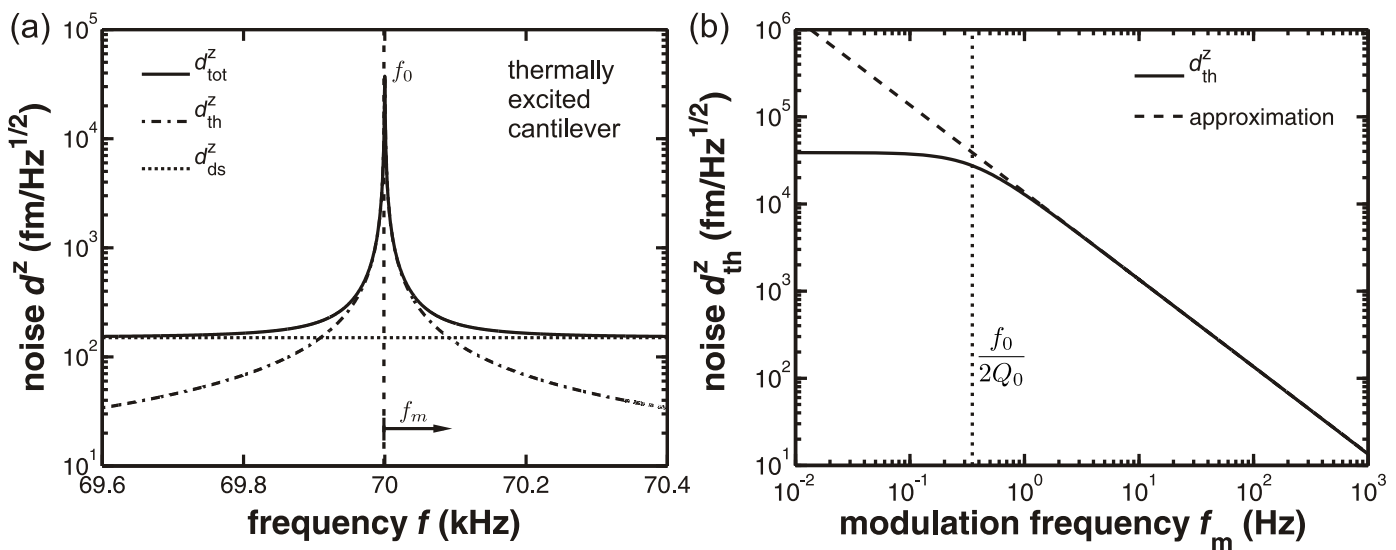

Figure 3: Illustrative representation of noise properties for a cantilever with $f_{0}=70 \mathrm{kHz}, k=2.5 \mathrm{~N} / \mathrm{m}$ and $Q_{0}=100000$ that is solely excited by its contact to a thermal bath at room temperature. (a) Calculated total-displacement noise spectral density $d_{\text {tot }}^{z}$ (solid line) compared to the thermal-noise contribution $d_{\mathrm{th}}^{z}$ (dash-dotted line) and the detection-system noise $d_{\mathrm{ds}}^{z}=150 \mathrm{fm} / \sqrt{\mathrm{Hz}}$ (dotted line). (b) Comparison between the thermal-displacement noise spectral density $d_{\mathrm{th}}^{z}\left(f_{0} \pm f_{m}\right)$ as given in Equation 2 (solid line) and the approximation of Equation 3 (dashed line) for the cantilever with a corner frequency of $f_{0} /\left(2 Q_{0}\right)=0.35 \mathrm{~Hz}$. Considering the oscillating cantilever as a mechanical low-pass filter for the displacement noise close to $f_{0}$, the corner frequency defines the point at which the noise is attenuated by $3 \mathrm{~dB}$. At modulation frequencies larger than the corner frequency, $d_{\mathrm{th}}^{Z}$ decreases essentially as $1 / f_{m}$ 
nents used in the detection system. In contrast to thermal noise, which is a fixed quantity for a given cantilever and temperature, the detection-system noise floor can be reduced by technical improvements of the detection system $[3,7,8]$.

\section{Frequency-shift noise}

The frequency demodulator of the NC-AFM system extracts the cantilever response to the tip-surface interaction from the sidebands of the cantilever-oscillation frequency spectrum (see Figure 2) and yields the signal power spectral density present in the sidebands, i.e., the displacement power spectral density $D^{z}(f)$ is transformed to the frequency-shift power spectral density $D^{\Delta f}\left(f_{m}\right)$ by the demodulation process. The noise contribution $D_{\text {tot }}^{\Delta f}\left(f_{m}\right)$ in this spectrum is the most relevant noise figure in NC-AFM measurements and can be calculated from the demodulator input noise by applying the appropriate demodulator transfer function and an approximation to obtain a simple yet accurate expression for the thermal-displacement power spectral density $D_{\mathrm{th}}^{z}$. As the frequency noise is represented as a function of the modulation frequency $f_{m}$, it is desirable to represent the displacement noise as a function of $f_{0} \pm f_{m}$. For $D_{\mathrm{th}}^{z}$, we use the following approximation [1] instead of the precise result from Equation 2:

$$
D_{\text {th }}^{z}\left(f_{0} \pm f_{m}\right) \simeq \frac{k_{\mathrm{B}} T f_{0}}{2 \pi k_{0} Q_{0}} \frac{1}{f_{m}^{2}}
$$

This expression is a very good approximation for modulation frequencies $f_{m}$ exceeding the cantilever corner frequency $f_{0} /\left(2 Q_{0}\right)$ as seen in Figure $3 \mathrm{~b}$. This approximation covers most of the practically relevant spectral range as the corner frequency is smaller than $1 \mathrm{~Hz}$ for high- $Q$ cantilevers. Combining Equation 1 and Equation 3 yields a simple yet accurate expression for the power spectral density of the total displacement noise in an FM-AFM system operated under high-Q conditions [4]:

$$
D_{\text {tot }}^{z}\left(f_{0} \pm f_{m}\right) \simeq \frac{k_{\mathrm{B}} T f_{0}}{2 \pi k_{0} Q_{0}} \frac{1}{f_{m}^{2}}+D_{\mathrm{ds}}^{z}
$$

To obtain the noise power spectral density of the frequencyshift signal present at the demodulator output, the demodulator amplitude response for noise $G_{\text {demod }}=\sqrt{2} f_{m} / A$ is applied, and we find [4]

$$
\widetilde{D}_{\text {tot }}^{\Delta f}=G_{\text {demod }}^{2} D_{\text {tot }}^{z}=\frac{f_{0} k_{\mathrm{B}} T}{\pi k_{0} Q_{0} A^{2}}+\frac{2 f_{m}^{2}}{A^{2}} D_{\mathrm{ds}}^{z} .
$$

As apparent from Equation 5, the contribution of the thermal noise to the total noise is independent of the modulation frequency $f_{m}$, whereas the detection-system-noise power contribution is amplified by the square of the modulation frequency. We further note that the total noise power in $\Delta f$ depends on the reciprocal of the squared cantilever oscillation amplitude.

The frequency-shift noise spectral density $\widetilde{d}_{\text {tot }}^{\Delta f}$ and its components as described in Equation 5 are shown as a function of the modulation frequency $f_{m}$ in Figure 4 for typical experimental conditions neglecting bandwidth limitations. This result clearly points to the experimental parameters determining the frequency-shift noise: the thermal limit is defined by the temperature $T$ and cantilever properties, namely the ratio $f_{0} /\left(k_{0} Q_{0}\right)$. For a cantilever with given $f_{0}$ and $k_{0}$, it is most important to yield a high effective $Q$-factor that may considerably differ from the intrinsic $Q$-factor [9] if one is interested in reducing the thermal-noise limit to the lowest possible value. The noise contribution from the detection system depends on the required bandwidth $B$ (range of $f_{m}$ ) and the quality of the detection system represented by $d_{\mathrm{ds}}^{z}$. Overall, $\widetilde{d}_{\text {tot }}^{\Delta f}$ scales with the inverse of the cantilever oscillation amplitude $A$. In Figure 4, the thermal noise limit is shown for typical cantilever properties and for $T=300 \mathrm{~K}$ as dash-dotted lines representing different $Q$-factors. From Figure 4 we can deduce the displacement noise floor of the detection system $d_{\mathrm{ds}}^{z}$ that must not be exceeded for a thermal-noise-limited measurement. We define the bandwidth $B_{\text {th }}^{\lim }$ for a thermal-noise-limited measurement by the frequency where the contributions of $d_{\mathrm{th}}^{z}$ and $d_{\mathrm{ds}}^{z}$ to the total frequency-shift noise spectral density $\widetilde{d}_{\text {tot }}^{\Delta f}$ are equal. This frequency $f_{\mathrm{m}}^{\lim }$ corresponds to the crossing point between the dashed and dash-dotted lines in Figure 4.

It follows that operation at the thermal noise limit can only be obtained if the bandwidth $B$ of the demodulator is set close to

$$
B_{\mathrm{th}}=\sqrt{\frac{f_{0} k_{\mathrm{B}} T}{2 \pi k_{0} Q_{0}}} \times \frac{1}{d_{\mathrm{ds}}^{z}}
$$

where the noise spectral density $d_{\mathrm{ds}}^{z}$ is treated here as a constant. This is fully justified by its white-noise character around the cantilever resonance. Note that this bandwidth limitation is solely based on noise considerations and does not reflect other bandwidth requirements, such as the stable operation of the PLL. However, there is a bandwidth limitation in any real system and $\widetilde{D}^{\Delta f}$ has to be considered as a hypothetical quantity that is rarely accessible. In any PLL system of practical use, the detection bandwidth is defined by internal filters, loop-gain settings and time constants that are normally accessible to the user for an optimisation of the signal processing. 


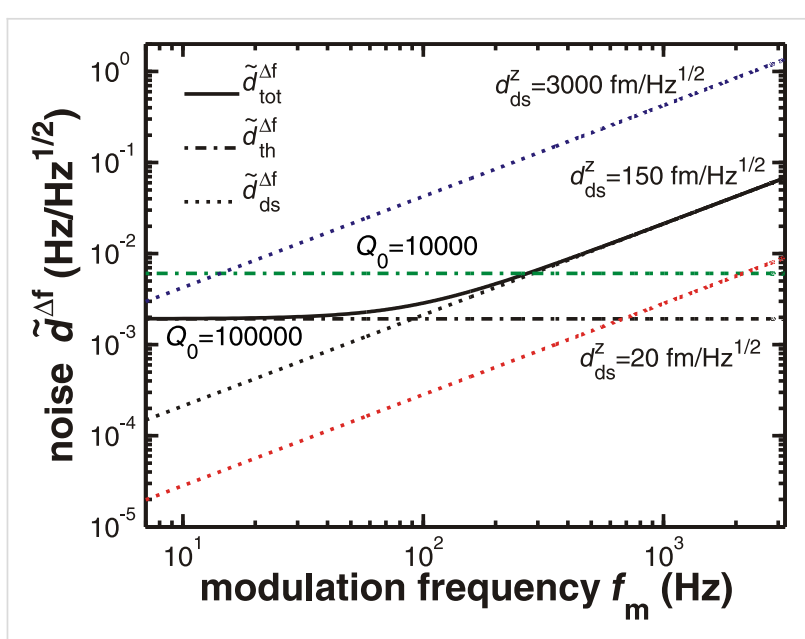

Figure 4: Illustrative representation of the noise spectral density for the total frequency-shift noise $\tilde{d}_{\text {tot }}^{\Delta f}=\sqrt{\tilde{D}_{\text {tot }}^{\Delta f}}$ for a system without bandwidth limitations. The total noise is composed of contributions from the thermal noise $\tilde{d}_{\mathrm{th}}^{\Delta f}=\sqrt{\tilde{D}_{\mathrm{th}}^{\Delta f}}$ plotted for different $Q$-factors and the noise of the frequency-shift detection system $\tilde{d}_{\mathrm{ds}}^{\Delta f}$ plotted for different values of the noise floor $d_{\mathrm{ds}}^{z}=\sqrt{D_{\mathrm{ds}}^{z}}$. Cantilever and oscillation parameters are $f_{0}=70 \mathrm{kHz}, k_{0}=2.5 \mathrm{~N} / \mathrm{m}$ and $A=10 \mathrm{~nm}$.

Thus, a complete PLL is modelled by using $G_{\mathrm{PLL}}=G_{\text {filter }} \times$ $G_{\text {demod }}$, with $G_{\text {filter }}$ being the amplitude response for the aforementioned filters (see Figure 1). Taking the amplitude response of the full PLL system into account, we obtain for the accessible noise power spectral density

$$
\begin{aligned}
D_{\mathrm{tot}}^{\Delta f} & =D_{\mathrm{th}}^{\Delta f}+D_{\mathrm{ds}}^{\Delta f} \\
& =G_{\text {filter }}^{2} \widetilde{D}_{\mathrm{tot}}^{\Delta f} \\
& =G_{\text {filter }}^{2}\left(\frac{f_{0} k_{\mathrm{B}} T}{\pi k_{0} Q_{0} A^{2}}+\frac{2 f_{m}^{2}}{A^{2}} D_{\mathrm{ds}}^{z}\right)
\end{aligned}
$$

at the output of the bandwidth-limited PLL system. The experimental determination of an unknown amplitude response $G_{\text {filter }}$ is described in Section 3 of Supporting Information File 1. To characterise the demodulator output noise with a single number, we define $\delta f_{\text {tot }}$ as the root mean square (RMS) of the overall frequency-shift noise:

$$
\delta f_{\text {tot }}=\left(\int_{0}^{\infty} D_{\text {tot }}^{\Delta f}\left(f_{m}\right) d f_{m}\right)^{1 / 2}
$$

where the integration can practically be limited to an upper frequency limit related to the detection bandwidth $B$. This is fully justified as filtering in the demodulator always yields a low-pass characteristic. A discussion of the RMS noise figure and its calculation by using approximations for the demodu- lator bandwidth is presented in Section 4 of Supporting Information File 1.

\section{Experimental}

Noise measurements are performed with three NC-AFM systems, named systems A, B and C in the following. All systems are well decoupled from mechanical vibrations by spring suspension and eddy-current damping systems. As an additional precaution, connections between the electronics and piezos are removed during noise measurements to ensure that measurements are not affected by any spurious electrical signals exciting the cantilever. All systems investigated here are based on the optical beam-deflection scheme for measuring the cantilever displacement. Therefore, the laser-light power $P_{\mathrm{pd}}$ reaching the photodetector is a parameter characterising the system. $P_{\mathrm{pd}}$ is calculated from the sum signal of the PSD, which in turn depends on the spectral sensitivity of the photodiode, the used laser light wavelength and the DC transimpedance of the preamplifier. The calibration of the detection system is described in Section 1 of Supporting Information File 1.

System A is a room-temperature UHV AFM/STM (Omicron NanoTechnology GmbH, Taunusstein, Germany) equipped with an easyPLL (Nanosurf AG, Liestal, Switzerland) for frequency demodulation. The AFM/STM setup has been modified by replacing the light source (light-emitting diode exchanged with a laser diode) and using optimised preamplifiers. Preamplifiers have been optimised for low-noise operation at frequencies around $100 \mathrm{kHz}$ and $300 \mathrm{kHz}$, respectively, and are exchanged depending on the eigenfrequency of the cantilever. Details on this modification and the frequency response of the preamplifiers can be found in [7]. The light source is a 48TE-SOT (Schäfter+Kirchhoff GmbH, Hamburg, Germany) and emits light at a wavelength of $685 \mathrm{~nm}$, while the PSD has a spectral sensitivity of $0.45 \mathrm{~A} / \mathrm{W}$ at this wavelength. Noise spectra are recorded with an SR770 spectrum analyser (Stanford Research Systems, Inc., Sunnyvale, CA, USA).

System B is a UHV VT AFM/STM (Omicron NanoTechnology $\mathrm{GmbH}$, Taunusstein, Germany) equipped with an easyPLL plus (Nanosurf AG, Liestal, Switzerland) as the demodulator. This system uses a light source having a wavelength of $830 \mathrm{~nm}$, while the spectral sensitivity of the PSD is $0.57 \mathrm{~A} / \mathrm{W}$ at this wavelength. Noise spectra are measured using the zoom FFT module of a HF2LI lock-in detector (Zurich Instruments AG, Zurich, Switzerland) for spectral analysis.

System C is a UHV 750 variable temperature STM/AFM with a PLLPro2 (software version 0.20.0) as the demodulator (RHK Technology, Inc., Troy, MI, USA). The light source is a laser source type 51 nanoFCM (Schäfter+Kirchhoff $\mathrm{GmbH}$, 
Hamburg, Germany) operated in the constant-power mode with radio-frequency modulation to reduce the coherence length to about $300 \mu \mathrm{m}$. The laser-light wavelength is $639 \mathrm{~nm}$, and a maximum output power of $5 \mathrm{~mW}$ is available at the fibre end while the PSD has a spectral sensitivity of $0.4 \mathrm{~A} / \mathrm{W}$ at this wavelength. A home-built preamplifier (low-bandwidth preamplifier) or the preamplifier supplied by the manufacturer (high-bandwidth preamplifier) is used depending on the bandwidth requirements. The frequency response of both preamplifiers is shown in Figure 5. To measure noise spectra, the SR770 spectrum analyzer (Stanford Research Systems, Inc., Sunnyvale, CA, USA) is used.

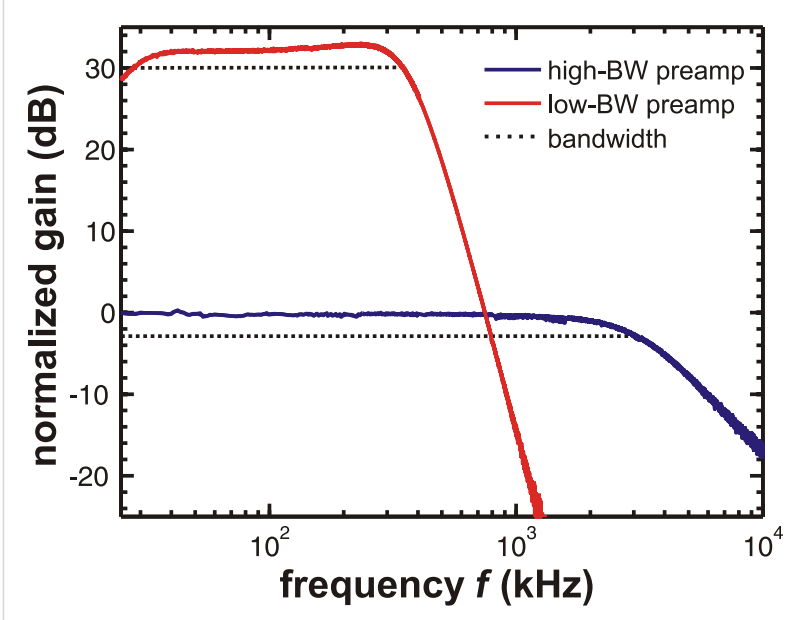

Figure 5: Frequency response of the high-bandwidth preamplifier (bandwidth $3.1 \mathrm{MHz}$ ) and the low-bandwidth preamplifier (bandwidth $320 \mathrm{kHz}$ ) for System C. The gain is normalised to the DC gain of the high-bandwidth preamplifier. Instead of connecting to the PSD, a sine wave of $0.5 \mathrm{~V}$ RMS amplitude was fed into a single quadrant input with a $100 \mathrm{k} \Omega$ resistor resulting in $5 \mu \mathrm{A}$ RMS current.

Force sensors are commercial silicon cantilevers (Nanoworld AG, Neuchâtel, Switzerland). For our investigations, we use a set of cantilevers covering a large range of resonance frequencies, $Q$-factors and stiffness, to explore the impact of these parameters on the noise figures. Cantilever specifications are compiled in Table 1 and Table 2 (cantilevers D and AO are type $\mathrm{FM}$, cantilevers $\mathrm{AQ}, \mathrm{AR}$ and $\mathrm{V}$ are type $\mathrm{NCH}$, cantilever $\mathrm{AP}$ is type $\mathrm{NCVH}$ and cantilever $\mathrm{AL}$ is type Arrow ${ }^{\mathrm{TM}}$ according to the commercial classification scheme).

The cantilever eigenfrequencies of the fundamental and the first and second harmonic mode are determined by measuring resonance curves and fitting the amplitude response function to the data as described in [9]. This procedure also yields quality factors $Q_{n}$, while the stiffness $k_{\mathrm{dim}}$ is calculated from cantilever dimensions and material properties [10] and used as a good approximation to the modal stiffness $k_{0}$ [12].
Table 1: Fundamental properties of the cantilevers used for noise analysis. Length $I$, mean width $\bar{w}$ and thickness $t$ are provided by the manufacturer. The stiffness $k_{\mathrm{dim}}$ is calculated from the cantilever dimensions [10]. Typical properties of a qPlus sensor are taken from [11] for comparison.

\begin{tabular}{lllll} 
cantilever & $I(\mu \mathrm{m})$ & $\bar{w}(\mu \mathrm{m})$ & $t(\mu \mathrm{m})$ & $k_{\text {dim }}(\mathrm{N} / \mathrm{m})$ \\
\hline AO 3 & 224 & 30 & 3.0 & $3.0 \pm 0.9$ \\
D 5 & 229 & 30 & 2.9 & $2.5 \pm 0.8$ \\
AR 17 & 127 & 27 & 3.6 & $26 \pm 5$ \\
V 15 & 125 & 26 & 3.7 & $29 \pm 6$ \\
AQ 10 & 123 & 29 & 4.5 & $60 \pm 10$ \\
AP 5 & 40 & 24 & 2.0 & $130 \pm 50$ \\
AL 3 & 35 & 42 & 0.7 & $9 \pm 3^{a}$ \\
qPlus & 2400 & 126 & 214 & 1800 \\
\hline
\end{tabular}

a Value provided by the manufacturer.

\section{Results and Discussion}

The noise analysis is performed in two steps. First, we measure the displacement noise spectral density $d_{\text {tot }}^{z}$ and, second, we investigate how it is propagated to the frequency-shift noise spectral density $d_{\text {tot }}^{\Delta f}$. The displacement noise is measured by a spectrum analyser connected directly to the output of the preamplifier (see Figure 1). The measurement range of the spectrum analyser is set to a few kilohertz around the cantilever resonance frequency to obtain high spectral resolution. The spectral density of the noise in the signal $V_{z}$ is measured and converted to the displacement-noise spectral density $d_{\text {tot }}^{z}$ in units of $\mathrm{fm} / \sqrt{\mathrm{Hz}}$ by the calibration procedure outlined in Section 1 of Supporting Information File 1. Figure 6 shows a representative result obtained with system $C$. The measured displacement noise spectral density is shown in Figure 6a (solid lines), together with the thermal noise contribution $d_{\text {th }}^{z}$ (dashdotted line) calculated from the given cantilever properties by using Equation 2. The noise floor of the detection system $d_{\mathrm{ds}}^{z}$ (dotted lines) is measured beside the resonance peak where thermal noise becomes negligible (solid and dashed lines are identical). For a study on how the noise of the detection system $d_{\mathrm{ds}}^{z}$ propagates through the demodulation system, different noise levels are artificially created by using white noise from a waveform generator DS345 (Stanford Research Systems, Inc., Sunnyvale, CA, USA) added to the displacement signal $V_{z}$. The curve with $d_{\mathrm{ds}}^{z}=108 \mathrm{fm} / \sqrt{\mathrm{Hz}}$ represents the noise floor of the setup while the other curves show artificially increased noise levels.

To measure the frequency-shift noise $d_{\text {tot }}^{\Delta f}$, the cantilever is excited to an oscillation with typically $10 \mathrm{~nm}$ amplitude and the spectrum analyser is connected to the output of the demodulator (see Figure 1) to measure the voltage noise in the $\Delta f$ signal. The demodulator is adjusted to zero mean frequency 

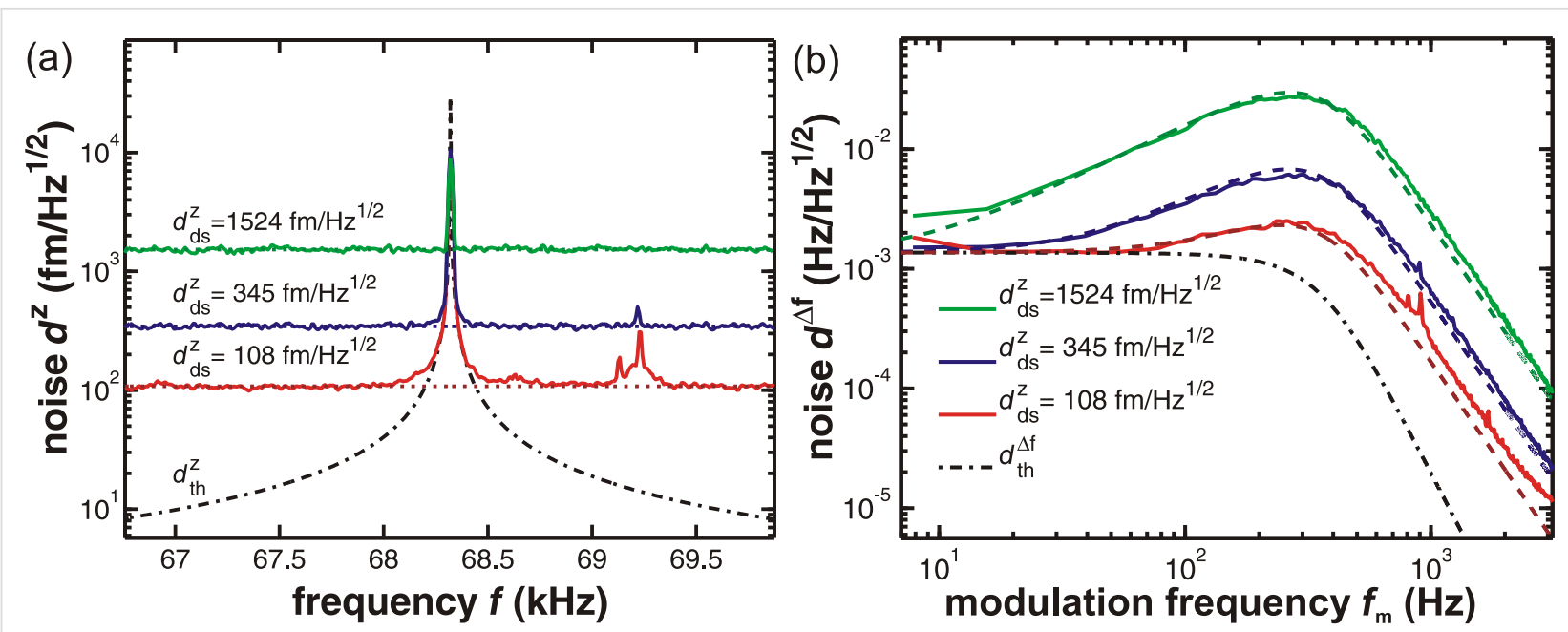

Figure 6: Measured and modelled noise figures for system C. (a) Different levels of displacement noise spectral density $d_{\text {tot }}^{z}$ at the output of the lowbandwidth preamplifier for a thermally excited cantilever. Solid lines represent measured data while dotted lines indicate the corresponding detectionsystem noise floor $d_{\mathrm{ds}}^{z}$. The dash-dotted line is a calculation of the displacement-thermal-noise spectral density $d_{\mathrm{th}}^{z}$ of the cantilever. (b) Calculated noise spectral density $d_{\text {tot }}^{\Delta f}$ at the PLL output for a cantilever oscillation amplitude of $5 \mathrm{~nm}$ and different noise floor levels (dashed lines) compared to measured data (solid lines). The dash-dotted line represents the modelled thermal noise contribution $d_{\mathrm{th}}^{\Delta f}$ to the noise in the $\Delta f$ signal. Measurements are performed with cantilever D 5 (see Table 1 and Table 2 for cantilever properties). Filter settings are $f_{c}=500 \mathrm{~Hz}, o=3, P=-2.0 \mathrm{~Hz} / \mathrm{deg}$ and $I=1 \mathrm{~Hz}$ (see Section 3 of Supporting Information File 1 for a detailed explanation).

shift and the measurement range of the spectrum analyser is set to the frequency region between 0 and $3 \mathrm{kHz}$. The measured voltage noise is multiplied by the known conversion factor of the demodulator (e.g., $30 \mathrm{~Hz} / \mathrm{V}$ ) to obtain the frequency-shiftnoise spectral density $d_{\text {tot }}^{\Delta f}$ in units $\mathrm{Hz} / \sqrt{\mathrm{Hz}}$. In Figure $6 \mathrm{~b}$, this quantity is shown for the same three levels of artificial detection-system displacement noise $d_{\mathrm{ds}}^{z}$ at the input of the demodulator as supplied for the measurement in Figure 6a. Measurements (solid lines) are compared to calculated curves (dashed lines) based on the $d_{\text {tot }}^{z}$ values obtained from the measurements shown in Figure 6a. The curve $d_{\mathrm{th}}^{\Delta f}\left(f_{m}\right)$ is determined from the cantilever properties and the filter settings of the PLL demodulator (thermal contribution in Equation 7) and represents the ideal case of the thermal noise of the cantilever without any detection-system noise. The trailing edge on the right side is caused by the attenuation through the low-pass filter with amplitude response $G_{\text {filter }}$ (see Section 3 of Supporting Information File 1 for details).

The dashed lines are model curves calculated using Equation 7 with the measured noise contribution $d_{\mathrm{ds}}^{z}$ and the calculated thermal noise contribution $d_{\mathrm{th}}^{z}$. The measured noise curves (solid lines) are in good agreement with the model (dashed lines). A disturbing side peak, which can be observed on top of $d_{\text {tot }}^{z}$ in Figure 6a for a low detection-system noise floor, similarly appears in the corresponding curve $d_{\text {tot }}^{\Delta f}$ in Figure 6b. Such peaks are due to electromagnetic emission from switching power supplies and other devices present in the laboratory environment. As the propagation of displacement noise $d_{\text {tot }}^{z}$ to frequency-shift noise $d_{\text {tot }}^{\Delta f}$ is well reproduced by experimental data, the latter can be used to obtain the level of the noise floor $d_{\mathrm{ds}}^{z}$ from a noise measurement in the low-frequency region of the $\Delta f$ signal. By inversion of Equation 7 , the displacementnoise spectral density $d_{\mathrm{ds}}^{z}=\sqrt{D_{\mathrm{ds}}^{z}}$ can be obtained from $D_{\text {tot }}^{\Delta f}$ if the system frequency response and $\widetilde{D}_{\mathrm{th}}^{\Delta f}$ are known:

$$
D_{\mathrm{ds}}^{z}=\frac{1}{G_{\text {demod }}^{2}}\left(\frac{D_{\mathrm{tot}}^{\Delta f}}{G_{\text {filter }}^{2}}-\widetilde{D}_{\mathrm{th}}^{\Delta f}\right) .
$$

At the frequency $f_{m}=f_{m}^{\max }$, where $f_{m} \times G_{\text {filter }}\left(f_{m}\right)$ has its maximum, the total noise is dominated by the noise from the detection system. At this point, Equation 9 can be simplified when assuming $D_{\text {th }}^{\Delta f} \ll D_{\text {tot }}^{\Delta f}$ yielding

$$
\begin{aligned}
D_{\mathrm{ds}}^{z} & \left.\approx \frac{D_{\text {tot }}^{\Delta f}}{G_{\mathrm{demod}}^{2} G_{\text {filter }}^{2}}\right|_{f_{m}=f_{m}^{\max }} \\
& \left.\approx \frac{A^{2} D_{\text {tot }}^{\Delta f}}{2 f_{m}^{2} G_{\text {filter }}^{2}}\right|_{f_{m}=f_{m}^{\max }} .
\end{aligned}
$$

This approximation defines an upper limit $d_{\mathrm{ds}, \max }^{z}=\sqrt{D_{\mathrm{ds}, \text { max }}^{z}}$ for the detection-system noise spectral density.

In this manner, we investigate the noise characteristics of the three NC-AFM systems using different preamplifiers and 
various cantilevers at different eigenmodes; the corresponding results are listed in Table 2. The detection noise measured directly in the displacement signal $V_{z}$ as shown in Figure 6a is denoted as $d_{\mathrm{ds}}^{z}$ while the same quantity obtained from the frequency-shift noise $d_{\text {tot }}^{\Delta f}$ by using Equation 9 is denoted as $d_{\mathrm{ds}, \Delta f}^{z}$. The upper limit derived from Equation 10 is denoted as $d_{\mathrm{ds}, \max }^{z}$. The latter is a useful approximation that can easily be calculated without knowledge of the cantilever properties.

Table 2 allows a comparison of the noise floor for different NC-AFM systems and demonstrates the influence of cantilever properties on the noise figures. The best values for the noise floor achieved here are around $100 \mathrm{fm} / \sqrt{\mathrm{Hz}}$ as measured for cantilever V 15 in system B and cantilevers D 5 and AR 17 in system $\mathrm{C}$. These cantilevers have a length in the range of
$100 \mu \mathrm{m}$ to $250 \mu \mathrm{m}$. Exchanging the preamplifier may cause a large difference in the noise floor. This can be observed for cantilevers AO 3 and AR 17 in system C where the noise floor is doubled by changing from the low-bandwidth to the highbandwidth preamplifier. The benefit of the high-bandwidth amplifier is the possibility to operate cantilevers at their higher resonance frequencies, where the displacement-noise floor significantly decreases even for a similar voltage noise caused by the laser-power-dependent photodiode shot noise due to different amplitude calibration factors for the corresponding modes. However, due to the length of only $40 \mu \mathrm{m}$ and $35 \mu \mathrm{m}$, for the high-frequency cantilevers AP 5 and AL 3, the laser adjustment becomes difficult, yielding only $77 \mu \mathrm{W}$ and $18 \mu \mathrm{W}$ laser power on the PSD compared to about $100 \mu \mathrm{W}$ for other cantilevers. Therefore, the detection-noise floor for these canti-

Table 2: Cantilever properties and noise figures for systems A, B and C. $f_{n}$ and $Q_{n}$ are the eigenfrequencies and $Q$-factors for the $n$th eigenmode of the cantilever. Noise-floor values $d_{\mathrm{ds}}^{z}$ are directly determined from the displacement signal $V_{z}$, while $d_{\mathrm{ds}, \Delta f}^{z}$ and $d_{\mathrm{ds}, \text { max }}^{z}$ are extracted from the $\Delta f$ noise at the demodulator output as described in the main text. $P_{\mathrm{pd}}$ is the total light power on the PSD. For system C, measurements are performed with two different preamplifiers. Missing $d_{d s}^{z}$ values are due to frequency-range limitations of the spectrum analyser. In the case of higher harmonics, we cannot easily calculate the modal cantilever stiffness, as it strongly depends on the tip mass, which is generally not known [13]. Therefore, determining $d_{\mathrm{ds}, \Delta f}^{z}$ requires the knowledge of the stiffness and is, thus, only calculated for the measurements at the fundamental resonance frequency. Typical properties of a system operated with a qPlus sensor are taken from [11] for comparison.

$\begin{array}{cccccc}\text { cantilever } \quad f_{n} & Q_{n} & d_{\mathrm{ds}}^{\mathrm{z}} & d_{\mathrm{ds}, \Delta f}^{\mathrm{z}} & d_{\mathrm{ds}, \max } & P_{\mathrm{pd}} \\ & (\mathrm{fm} / \sqrt{\mathrm{Hz}}) & (\mathrm{fm} / \sqrt{\mathrm{Hz}}) & (\mathrm{fm} / \sqrt{\mathrm{Hz}}) & (\mu \mathrm{W})\end{array}$

System A

\begin{tabular}{|c|c|c|c|c|c|c|}
\hline$A Q 10$ & $f_{0}=361,599 \mathrm{~Hz}$ & $Q_{0}=21,200$ & & 275 & 278 & 97 \\
\hline \multicolumn{7}{|c|}{ System B } \\
\hline$\vee 15$ & $f_{0}=279,451 \mathrm{~Hz}$ & $Q_{0}=47,200$ & 125 & 119 & 124 & 105 \\
\hline \multicolumn{7}{|c|}{ System C, low-bandwidth preamplifier } \\
\hline D 5 & $f_{0}=68,353 \mathrm{~Hz}$ & $Q_{0}=118,000$ & 115 & 122 & 130 & 120 \\
\hline AO 3 & $f_{0}=68,183 \mathrm{~Hz}$ & $Q_{0}=173,700$ & 237 & 223 & 226 & 106 \\
\hline AR 17 & $f_{0}=276,360 \mathrm{~Hz}$ & $Q_{0}=39,200$ & & 97 & 98 & 120 \\
\hline
\end{tabular}

System C, high-bandwidth preamplifier

\begin{tabular}{|c|c|c|c|c|c|}
\hline AO 3 & $f_{0}=68,183 \mathrm{~Hz}$ & $Q_{0}=173,700$ & 416 & 417 & 105 \\
\hline AO 3 & $f_{1}=437,086 \mathrm{~Hz}$ & $Q_{1}=48,500$ & & 93 & 105 \\
\hline $\mathrm{AO} 3$ & $f_{2}=1,235,138 \mathrm{~Hz}$ & $Q_{2}=15,200$ & & 51 & 105 \\
\hline AR 17 & $f_{0}=276,360 \mathrm{~Hz}$ & $Q_{0}=39,200$ & 258 & 259 & 120 \\
\hline AR 17 & $f_{1}=1,730,811 \mathrm{~Hz}$ & $Q_{1}=6,300$ & & 99 & 120 \\
\hline AP 5 & $f_{0}=1,996,199 \mathrm{~Hz}$ & $Q_{0}=32,400$ & 302 & 309 & 77 \\
\hline AL 3 & $f_{0}=1,316,757 \mathrm{~Hz}$ & $Q_{0}=16,600$ & 845 & 892 & 18 \\
\hline
\end{tabular}

qPlus system

qPlus $\quad f_{0}=32,768 \mathrm{~Hz} \quad Q_{0}=5,000 \quad 62$


levers is much higher than for larger cantilevers. With an improved laser-spot adjustment, however, a noise floor close to $100 \mathrm{fm} / \sqrt{\mathrm{Hz}}$ should be possible.

In Figure 7, we illustrate the choice of optimum filter settings for a thermal-noise-limited detection. For that purpose, the frequency noise originating from the same detection system but passed through different PLL filters is shown. As these measurements are performed in system $\mathrm{C}$, the filters are modelled as a closed loop where the settings of the PI controller have a significant effect on the frequency response and need to be individually adjusted for each setting of the loop filter order $o$ and cutoff frequency $f_{c}$ (see Section 3 of Supporting Information File 1 for details). The optimum settings for each loop filter used in the following are listed in Table S4 in Section 3 of Supporting Information File 1. In Figure 7a, we display the noise spectral characteristics of the $\Delta f$ signal, while Figure $7 \mathrm{~b}$ shows a plot of the total noise represented by the RMS value of the $\Delta f$ signal as a function of the cantilever oscillation amplitude. Using a bandwidth of $B_{-3 \mathrm{~dB}}=385 \mathrm{~Hz}\left(f_{c}=1 \mathrm{kHz}\right.$, $o=5$ ), the total noise exceeds the thermal noise level by half an order of magnitude. Choosing a much lower bandwidth of $B_{-3 \mathrm{~dB}}=48 \mathrm{~Hz}\left(f_{c}=125 \mathrm{~Hz}, o=5\right)$ decreases the frequency range where the signal is not attenuated below the one defined by the thermal noise limit. The optimum filter setting for the $d_{\mathrm{ds}}^{z}$ noise floor present in this measurement is a filter setting with a bandwidth of $B_{-3 \mathrm{~dB}}=103 \mathrm{~Hz}\left(f_{c}=125 \mathrm{~Hz}, o=1\right)$, where the total noise does not significantly exceed the thermal noise and the signal is not unnecessarily attenuated. For all filter settings investigated here, experiment (solid lines) and model (dashed lines) agree well with each other.

In Figure $7 \mathrm{~b}$, measured values $\delta f_{\text {tot }}$ (circles, squares, triangles) are compared to calculated values $\delta f_{\text {tot }}$ derived from Equation 8 (solid lines) and $\delta f_{\text {th }}$ defining the thermal limit of the RMS frequency-shift noise (dash-dotted lines). While there is a large difference between thermal noise and total noise for the largebandwidth filter setting $\left(f_{c}=1 \mathrm{kHz}, o=5\right)$, this discrepancy becomes smaller and finally negligible on further reduction of the bandwidth. Note, however, that the settings yielding the smallest RMS noise are not the optimum as the corresponding filter does not only reduce the noise but attenuates the NC-AFM signal more than necessary for thermal-noise-limited operation.

The RMS value of the total noise is an important figure of merit of the NC-AFM detection system, as it defines the minimum detectable frequency shift. Figure $7 \mathrm{~b}$ is an excellent demonstration of the potential of small amplitudes for atomic resolution measurements as it is known that the atomic contrast increases with reduced cantilever oscillation amplitude $[11,14]$. For a measurement with $B_{-3 \mathrm{~dB}}=385 \mathrm{~Hz}$ (red line, $f_{c}=1 \mathrm{kHz}, o=5$ ), one would choose an amplitude of $5 \mathrm{~nm}$ or above to reduce the noise; however, this would also reduce the atomic contrast compared to a lower amplitude measurement. For a measurement with $B_{-3 \mathrm{~dB}}=103 \mathrm{~Hz}$ (blue line, $f_{c}=125 \mathrm{~Hz}, o=1$ ), one can take full advantage of the increased atomic corrugation for the smaller amplitude, as the total noise is even below the thermal noise for the larger amplitude.
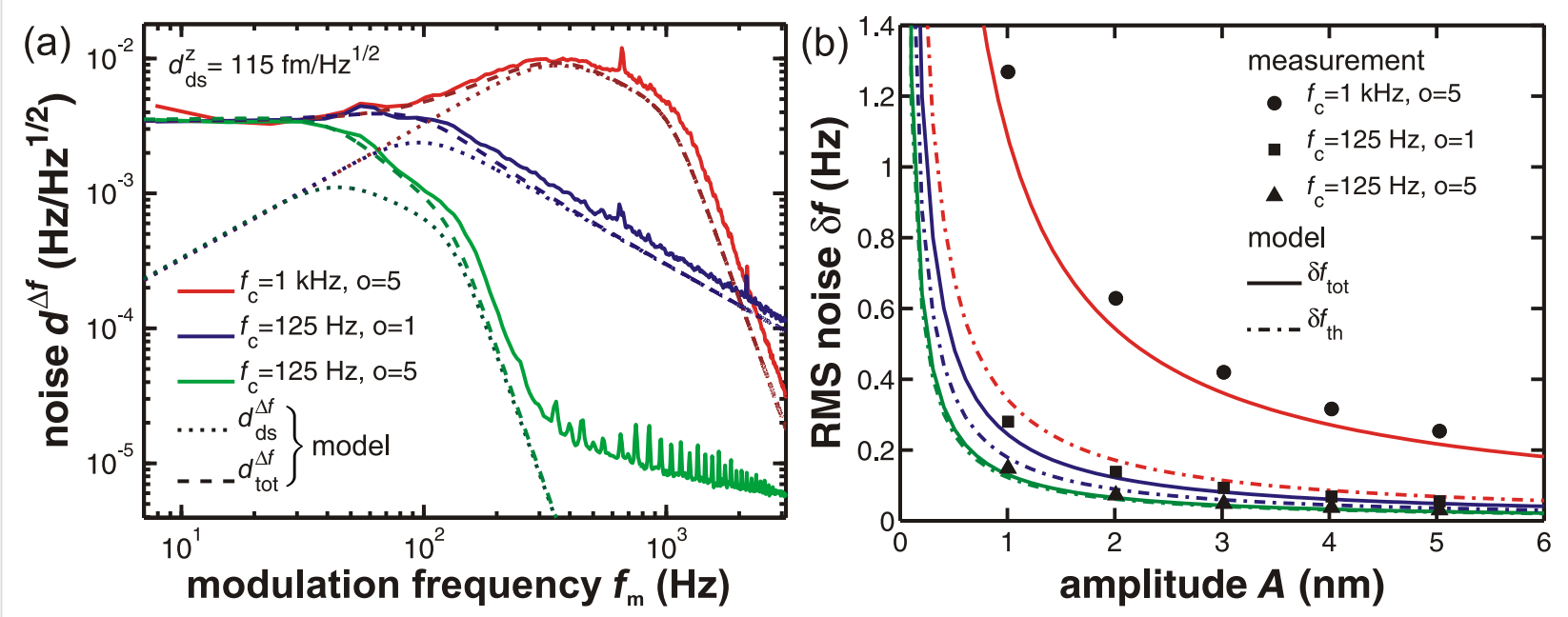

Figure 7: (a) Measured (solid) and modelled (dashed) frequency-shift-noise spectral density $d_{\text {tot }}^{\Delta f}$ using three different filter settings. Dotted lines show the contribution $d_{\mathrm{ds}}^{\Delta f}$ of the detection-system noise to the total frequency-shift-noise spectral density for a noise floor of $d_{\mathrm{ds}}^{z}=115 \mathrm{fm} / \sqrt{\mathrm{Hz}}$ at the input of the demodulator. The oscillation amplitude is $5 \mathrm{~nm}$. (b) Noise figures for different cantilever oscillation amplitudes. Measured RMS frequency-shift noise $\delta f$ (circles, squares, triangles) for different PLL filter settings compared to predictions from Equation 8 (solid lines) using the measured detection-system noise $d_{\mathrm{ds}}^{z}$ in the cantilever displacement signal $V_{z}$. Dash-dotted lines represent calculations of the thermal-noise contribution $\delta f_{\text {th }}$. Measurements are performed with cantilever D 5 in system C (see Table 1 and Table 2 for cantilever properties). 

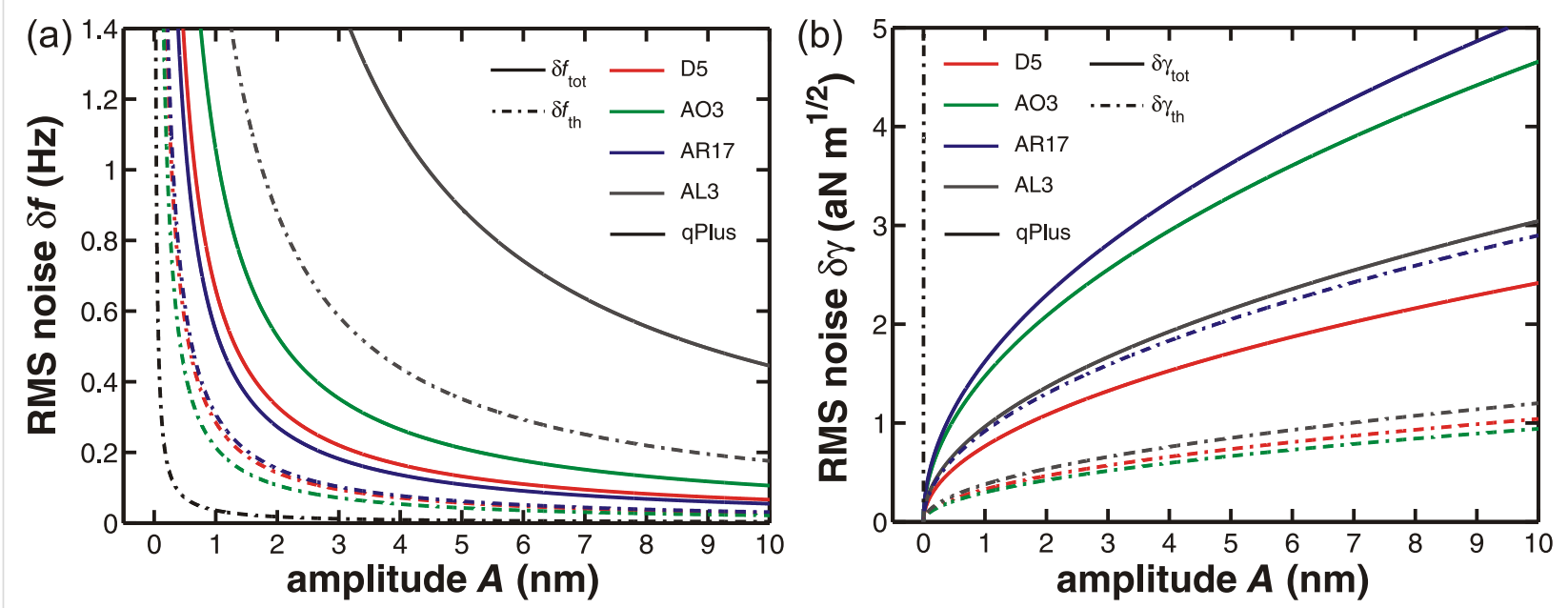

Figure 8: (a) RMS frequency-shift noise $\delta f$ and (b) normalised RMS frequency-shift noise $\delta y$ in the limit of purely thermal noise (dash-dotted lines) as well as in combination with the corresponding detection noise (solid lines) for different cantilevers $\left(T=300 \mathrm{~K}\right.$, filter settings $f_{C}=500 \mathrm{~Hz}, o=3$, $P=-2.0 \mathrm{~Hz} / \mathrm{deg}$ and $I=1 \mathrm{~Hz}$ yielding $B_{-3 \mathrm{~dB}}=258 \mathrm{~Hz}$ ). Calculations are performed for the fundamental eigenfrequency $f_{0}$ of the cantilever. Cantilever properties and the corresponding values of the displacement-noise floor of the detection system $d_{\mathrm{ds}}^{z}$ are given in Table 1 and Table 2.

In Figure 8a, different cantilevers are compared regarding their total RMS frequency shift noise $\delta f_{\text {tot }}$ (solid lines) as well as the thermal frequency noise $\delta f_{\text {th }}$ (dash-dotted lines). Here, the same bandwidth of $B_{-3 \mathrm{~dB}}=258 \mathrm{~Hz}$ is chosen for all simulations to facilitate the comparison of the cantilevers. Regarding thermal noise, all cantilevers except AL 3 exhibit an RMS noise below $0.5 \mathrm{~Hz}$ for amplitudes larger than $1 \mathrm{~nm}$. The total noise values are ordered by the level of the corresponding noise floor $d_{\mathrm{ds}}^{z}$, dominating the total noise for a bandwidth larger than the thermal-limit bandwidth. Note that the thermal-noise contribution of AL 3 is even larger than the total noise of the other cantilevers. These results are compared to typical values for a qPlus sensor with parameters taken from [11]. The thermal noise $\delta f_{\text {th }}$ of the qPlus sensor is an order of magnitude below the values for the cantilevers. Including the noise of the detection system, $\delta f_{\text {tot }}$ of the qPlus sensor is nearly identical to the thermal noise $\delta f_{\text {th }}$ obtained for cantilever D 5 (curve not shown) and, therefore, only half of the noise level of the best cantilevers.

For a valid comparison of measurements obtained under different experimental conditions, however, it is important to compare limits in the normalised frequency shift $\gamma$ rather than the plain frequency shift $\Delta f$. Based on the concept of the normalised frequency shift [15], we define a normalised-frequencyshift RMS noise as

$$
\delta \gamma=k A^{3 / 2} \delta f / f_{0}
$$

to compare the noise characteristics of cantilevers independently of their stiffness and resonance frequency and display the corresponding data as a function of the cantilever oscillation amplitude in Figure 8b. Regarding the thermal contribution $\delta \gamma_{\text {th }}$ to the normalised frequency-shift noise, cantilevers D 5 and AO 3 exhibit the best performance but are closely followed by cantilever AL 3. The $\delta \gamma_{\text {th }}$ value of AR 17 is even larger than the total noise $\delta \gamma_{\text {tot }}$ of cantilever D 5. This is presumably due to the large ratio $k / f_{0}$. Although cantilever AL 3 has the largest detection-system noise floor, its $\delta \gamma_{\text {tot }}$ is quite close to that of cantilever D 5. On the other hand, the qPlus sensor has a noise level $\delta \gamma$ more than two orders above the results for the cantilevers due to its exceptional $k / f_{0}$ ratio. Therefore, the advantageous noise figures of the qPlus sensor documented in Figure 8a can only be exploited if the sensor is operated at very low amplitudes.

As cantilevers D 5 and AL 3 have thermal bandwidth limits of $B_{\text {th }}^{\lim }=95 \mathrm{~Hz}$ and $B_{\text {th }}^{\lim }=85 \mathrm{~Hz}$ according to Equation 6, they are best suited for thermal-noise-limited operation. Operating them with a filter $B_{-3 \mathrm{~dB}}=103 \mathrm{~Hz}\left(f_{c}=125 \mathrm{~Hz}, o=1\right)$ yields noise limits of $\delta \gamma_{\text {tot }}=0.69 \mathrm{aN} \sqrt{m}$ and $\delta \gamma_{\text {tot }}=0.84 \mathrm{aN} \sqrt{m}$, respectively, for an oscillation amplitude of $A=5 \mathrm{~nm}$. Assuming, the detection noise floor of AL 3 could be decreased to $130 \mathrm{fm} / \sqrt{\mathrm{Hz}}$ as for cantilever D 5, thermal-noise-limited operation with a bandwidth of $B_{\mathrm{th}}^{\lim }=586 \mathrm{~Hz}$ and $\delta \gamma_{\mathrm{tot}}=1.62 \mathrm{aN} \sqrt{m}$ would be possible for an oscillation amplitude of $5 \mathrm{~nm}$ and a filter setting of $B_{-3 \mathrm{~dB}}=646 \mathrm{~Hz}\left(f_{c}=1000 \mathrm{~Hz}, o=3\right)$. This means that by switching from cantilever D 5 to AL 3, the usable bandwidth could be increased by a factor of six at the cost of 
increasing $\delta \gamma_{\text {tot }}$ by a factor of two. In comparing such numbers, one should, however, consider that the assumed oscillation amplitude of $5 \mathrm{~nm}$ may be at the limit of stable operation [15], specifically for the soft cantilever D 5. In conclusion, the highfrequency and relatively stiff cantilever AL 3 represents an excellent choice for high-speed measurements with small amplitudes and good noise performance, while the larger and softer cantilever D 5 is the better choice for slower measurements with best possible noise performance.

\section{Conclusion}

We investigated the relation between the displacement noise in NC-AFM measurements and the corresponding frequency-shift noise at the output of the demodulator and demonstrated that predictions based on the demodulator transfer function and filtering are well reproduced by experiments. For a quantitative analysis of the noise, a precise amplitude calibration of the detection system relating electrical signals to the mechanical oscillation of the cantilever is inevitable. The displacement noise of an NC-AFM system can be measured directly with a spectrum analyser at the output of the detection system, and the thermal component of the displacement noise extracted from such spectra agrees well with spectra derived from a model of thermal cantilever excitation. The noise contribution of the detection system can be obtained from the white-noise floor of the measured spectra. The knowledge of the detection-system transfer functions allows one to predict the frequency-shift noise from the measured displacement noise, and by inversion, a measurement of the detection-system noise from the frequency-shift noise is possible. While the former analysis requires a spectrum analyser with very high resolution and an operating range that includes the eigenfrequency of the cantilever, the latter procedure requires only a measurement of the frequency-shift noise with a device covering the frequency range between a few hertz and about $10 \mathrm{kHz}$ at moderate frequency resolution. Therefore, a rather complete noise characterisation with a simple spectrum analyser as integrated in many NC-AFM systems is possible for a calibrated system.

The framework of modelling noise in the NC-AFM system in combination with the experimental practice described here provides a clear guideline for system design and the choice of experimental parameters for thermal-noise-limited operation. The analysis shows that for a noise-optimised NC-AFM measurement, the right choice of the cantilever is most important, and obtaining a high effective $Q$-factor should be given great attention to keep the level of thermal noise at a minimum. The bandwidth of thermal-noise-limited operation is determined by the noise generated in the detection system. By an appropriate choice of PLL filter settings, one can make full use of this bandwidth without attenuating the NC-AFM signal while very effi- ciently eliminating most of the detection system noise. We find that with a technically optimised system and an appropriate choice of experimental parameters, room-temperature thermalnoise-limited NC-AFM measurements are possible over a bandwidth of $100 \mathrm{~Hz}$ and a detection limit smaller than $0.7 \mathrm{aN} \sqrt{m}$ for the normalised frequency shift operating at an amplitude of $5 \mathrm{~nm}$.

\section{Supporting Information}

\section{Supporting Information File 1}

Experimental details and theory.

[http://www.beilstein-journals.org/bjnano/content/

supplementary/2190-4286-4-4-S1.pdf]

\section{Acknowledgements}

The authors gratefully acknowledge experimental support from Stefan Kuhn and Hans-Hermann Pieper and most helpful discussions with Steffen Porthun, Sadik Hafizovic, Kei Kobayashi and Hirofumi Yamada. Substantial help from Alfred Ziegler with the mathematics of Section 2 in Supporting Information File 1 is gratefully acknowledged. The project has been generously supported by Nanoworld Services GmbH.

\section{References}

1. Albrecht, T. R.; Grütter, P.; Horne, D.; Rugar, D. J. Appl. Phys. 1991, 69, 668-673. doi:10.1063/1.347347

2. Kobayashi, K.; Yamada, H.; Itoh, H.; Horiuchi, T.; Matsushige, K. Rev. Sci. Instrum. 2001, 72, 4383-4387. doi:10.1063/1.1416104

3. Fukuma, T.; Kimura, M.; Kobayashi, K.; Matsushige, K.; Yamada, H. Rev. Sci. Instrum. 2005, 76, 053704. doi:10.1063/1.1896938

4. Kobayashi, K.; Yamada, H.; Matsushige, K. Rev. Sci. Instrum. 2009, 80, 043708. doi:10.1063/1.3120913

5. Polesel-Maris, J.; Venegas de la Cerda, M. A.; Martrou, D.; Gauthier, S. Phys. Rev. B 2009, 79, 235401. doi:10.1103/PhysRevB.79.235401

6. Lübbe, J.; Temmen, M.; Schnieder, H.; Reichling, M. Meas. Sci. Technol. 2011, 22, 055501. doi:10.1088/0957-0233/22/5/055501

7. Torbrügge, S.; Lübbe, J.; Tröger, L.; Cranney, M.; Eguchi, T.; Hasegawa, Y.; Reichling, M. Rev. Sci. Instrum. 2008, 79, 083701. doi:10.1063/1.2964119

8. Rode, S.; Stark, R.; Lübbe, J.; Tröger, L.; Schütte, J.; Umeda, K.; Kobayashi, K.; Yamada, H.; Kühnle, A. Rev. Sci. Instrum. 2011, 82, 073703. doi:10.1063/1.3606399

9. Lübbe, J.; Tröger, L.; Torbrügge, S.; Bechstein, R.; Richter, C.; Kühnle, A.; Reichling, M. Meas. Sci. Technol. 2010, 21, 125501. doi:10.1088/0957-0233/21/12/125501

10. Lübbe, J.; Doering, L.; Reichling, M. Meas. Sci. Technol. 2012, 23, 045401. doi:10.1088/0957-0233/23/4/045401

11. Giessibl, F. J.; Pielmeier, F.; Eguchi, T.; An, T.; Hasegawa, Y. Phys. Rev. B 2011, 84, 125409. doi:10.1103/PhysRevB.84.125409

12. Lübbe, J.; Temmen, M.; Rahe, P.; Kühnle, A.; Reichling, M. Beilstein J. Nanotechnol. 2013, submitted. 
13. Melcher, J.; Hu, S.; Raman, A. Appl. Phys. Lett. 2007, 91, 053101. doi:10.1063/1.2767173

14. Giessibl, F. J.; Bielefeldt, H.; Hembacher, S.; Mannhart, J. Appl. Surf. Sci. 1999, 140, 352-357. doi:10.1016/S0169-4332(98)00553-4

15. Giessibl, F. J. Phys. Rev. B 1997, 56, 16010-16015 doi:10.1103/PhysRevB.56.16010

\section{License and Terms}

This is an Open Access article under the terms of the Creative Commons Attribution License

(http://creativecommons.org/licenses/by/2.0), which permits unrestricted use, distribution, and reproduction in any medium, provided the original work is properly cited.

The license is subject to the Beilstein Journal of Nanotechnology terms and conditions:

(http://www.beilstein-journals.org/bjnano)

The definitive version of this article is the electronic one which can be found at: doi:10.3762/bjnano.4.4 